\title{
Correction to: Finite-Velocity Diffusion in Random Media
}

\author{
Manuel O. Cáceres ${ }^{1,2}$ \\ Published online: 30 August 2020 \\ ๑) Springer Science+Business Media, LLC, part of Springer Nature 2020
}

\section{Correction to: Journal of Statistical Physics (2020) 179:729-747 https://doi.org/10.1007/s10955-020-02553-9}

In Eqs. (8) and (B10) exponential $e^{i k y_{1}}$ should be deleted, only the last one should remain as $e^{-i k y_{n}}$. This typo mistake does not modify any result in the paper.

In Eq. (C1) the exponential should be $e^{-i k y_{p}}$. This typo mistake does not modify any result in the paper.

After Eq. (B5) exponential should change sing, that is $e^{-i k x}$. This typo mistake does not modify any result in the paper.

In Eqs. (22) and (B14) exponential should change sing, that is $e^{-i k y_{1}}$. This typo mistake does not modify any result in the paper.

In Eq. (28) the expression for $\mathcal{D}_{E}(k, s)$ has an exponent -1 which should be deleted. This typo mistake does not modify any result in the paper.

In Sects. 2.1 and 3 the expression exact result should be quoted: dominant result. This is so because the $n$-fold integral (8) is not space ordered, thus there are contributions from overlapping domains. The proof that these domains can be discarded will be given elsewhere. This fact does not modify any result concerning EMA, which remain entirely true.

Publisher's Note Springer Nature remains neutral with regard to jurisdictional claims in published maps and institutional affiliations.

The original article can be found online at https://doi.org/10.1007/s10955-020-02553-9.

$凶$ Manuel O. Cáceres

caceres@cab.cnea.gov.ar

1 Comision Nacional de Energia Atomica, Centro Atomico Bariloche and Instituto Balseiro, Universidad Nacional de Cuyo, Av. E. Bustillo 9500, CP 8400 Bariloche, Argentina

2 CONICET, Centro Atomico Bariloche, Av. E. Bustillo 9500, CP 8400 Bariloche, Argentina 